\title{
N-Terminal Pro-B-Type Natriuretic Peptide as a Predictor of Adverse Outcomes After Off-Pump Coronary Artery Bypass Surgery: A Prospective Study
}

\author{
Mohamed Fawzy, Abdel-Aleem, MD,${ }^{1}$ Ibrahim Abobakr Elsedeeq, MSc, ${ }^{2}$ Gamal Hamid Ahmed, MD, ${ }^{1}$ \\ Tarek El-Tawil, MD, ${ }^{2}$ Amal Rizk, MD, ${ }^{1}$ Hesham Alkady, $\mathrm{MD}^{2}$ \\ ${ }^{1}$ Department of Cardiothoracic Surgery, Faculty of Medicine, Cairo University, Egypt; ${ }^{2}$ Department of Critical Care Medicine, \\ Faculty of Medicine, Cairo University, Egypt
}

\section{ABSTRACT}

Background: The strength of association between preoperative natriuretic peptide levels and adverse outcomes after cardiac surgery recently has been studied in different research, but results still are diversely variable.

Methods: Sixty-five consecutive patients undergoing elective off-pump coronary artery bypass grafting prospectively were recruited. Preoperative levels of NTproBNP were measured in venous blood samples collected before induction of anesthesia.

Results: The average age was $57.62 \pm 7.21$. Of the patients, $86.15 \%$ were male. Euro-scoreII averaged $1.76 \pm 0.34$. The mean preoperative NTproBNP levels were $312.41 \pm 329.93$ $\mathrm{pg} / \mathrm{mL}$. Only two patients died (3\%). Three patients required prolonged mechanical ventilation (4.6\%). Four patients $(6 \%)$ suffered from new onset postoperative AF. Five patients (7.6\%) had low cardiac output, of which three needed IABP, and four patients $(6 \%)$ had postoperative myocardial infarction. The mean ICU stay was $3.37 \pm 0.84$ days, and mean hospital stay was $6.38 \pm 1.3$ days. There were no significant differences in preoperative NTproBNP levels in patients who had or didn't have any of the postoperative complications or in-hospital mortality $(P>.05)$.

Conclusion: Our study showed no significant correlation between preoperative NTproBNP levels and postoperative low cardiac output, atrial fibrillation, postoperative myocardial infarction, length of ICU stay, prolonged mechanical ventilation, length of hospital stay as well as in-hospital mortality following elective off-pump CABG. Therefore, more prospective specific studies are needed to delineate the role of preoperative natriuretic peptides as significant predictors of poor outcome after CABG surgery.

\section{INTRODUCTION}

Natriuretic peptides (NPs) are produced in both atria and ventricles. Their levels in plasma, together with their inactive byproduct $\mathrm{N}$-terminal pro-B-type natriuretic peptide

Received Fuly 18, 2021; accepted August 10, 2021.

Correspondence: Hesham Alkady, Kasralaini str., Almanial, Cairo, Egypt; 00201002059723 (e-mail: heshamalqady@cu.edu.eg).
(NTproBNP), increase in failing ventricular myocardium in response to myocardial wall stretch [Iwanaga 2006]. The physiologic actions of NPs reduce cardiac preload and afterload to counteract the detrimental effects of pressure and volume overload seen in heart failure (HF). These physiologic processes are modulated by the neurohormonal counterregulatory system [Emdin 2004]. Therefore, atrial natriuretic peptide (ANP) and blood natriuretic peptide (BNP) levels reflect HF severity with different cardiovascular diseases and their concentrations were found to be independent risk markers for morbidity and mortality even more than left ventricular ejection fraction (EF) and New York Heart Association (NYHA) class [Gaggin 2014].

In patients undergoing cardiac surgery, accurate identification of high-risk patients is of paramount importance in order to implement prophylactic interventions reducing postoperative adverse outcomes. Although many clinical prognostic models such as EuroSCORE exist, most of these parameters are only useful in predicting mortality but not adverse events such as atrial fibrillation (AF) and cardiogenic shock requiring intraaortic balloon pulsations (IABP). This why the strength of association between preoperative natriuretic peptide levels and adverse outcomes after cardiac surgery recently has been studied in different research but results still are diversely variable [Litton 2012]. In this work, we study the role of preoperative NTproBNP level as a predictor of adverse postoperative outcomes and in-hospital mortality in a homogenous cohort of low-risk patients registered for elective OPCAB surgery, eliminating as much as possible factors that might influence such a predictive role.

\section{METHODS}

Preoperative data: In this prospective study, we recruited 65 adult patients registered for elective off-pump coronary artery bypass grafting (OPCAB) at our university hospitals over a period of one year. This study was approved by the institutional review board of our university, and informed written consent was signed by all patients for both withdrawal of samples and enrollment in the study. Patients were excluded if they had significant valvular heart disease requiring surgical intervention, dilated or hypertrophic cardiomyopathy, NYHA class III or IV, EF $<40 \%$, inotropic support or intra-aortic balloon pump before surgery, preoperative atrial 
Table 1. Preoperative characteristics of patients

\begin{tabular}{lc}
\hline Variables & Patients $(\mathrm{N}=65)$ \\
\hline $\begin{array}{l}\text { Age in years (mean } \pm \text { SD) } \\
\text { Gender }\end{array}$ & $57.62 \pm 7.21$ \\
Male & \\
Female & $56(86.15 \%)$ \\
Comorbidities & $9(13.85 \%)$ \\
Diabetes mellitus & \\
Hypertension & $10(15.38 \%)$ \\
Carotid artery stenosis & $42(64.62 \%)$ \\
Ejection Fraction (mean \pm SD) & $1(1.54 \%)$ \\
EuroScore II (mean \pm SD) & $50.91 \pm 8.13$ \\
& $1.76 \pm 0.34$
\end{tabular}

fibrillation, renal impairment in the form of creatinine clearance $<60 \mathrm{ml} / \mathrm{min}$, hyper-or hypo-thyroidism, and moderate to severe chronic obstructive pulmonary disease (COPD) determined by a forced expiratory volume in the 1st second (FEV1) $<80 \%$ of predicted, or continuous use of bronchodilators for $>2$ weeks. Beta-blocking agents and statins were given to all patients until the morning of surgery. Venous blood samples for measuring NT-proBNP levels were collected on the day of surgery before induction of anesthesia. Samples were sent to our university clinical pathology department to be stored in a deep freezer until measured. We used ELISA immunoassay technique that allows in vitro quantitative determination of human NTproBNP concentrations. ELISA SET (Tecan) machine was used for reading. A curve was plotted with a serial standard dilution log graph, plotting the mean absorbance for each standard on the $\mathrm{X}$-axis against the concentration on the Y-axis and drawing a best fit curve through the points on the graph. Finally, the concentration of NTproBNP in the sample was determined by comparing the OD (optical density) of the sample with the standard curve. The serum normal value of NTproBNP, according to our laboratory, is less than $120 \mathrm{pg} / \mathrm{mL}$ for patients aged $0-70$ years. All samples were blindly analyzed. Laboratory technicians were blinded to the clinical conditions of the included patients, and all clinicians involved in the patient medical care whether in the intensive care unit (ICU) or in-patient ward were blinded to the preoperative NTproBNP sample results.

Operative techniques: All operations were performed through a full median sternotomy. All patients underwent multi-vessel OPCAB surgery with anastomosing the left internal mammary artery (LIMA) to the left anterior descending (LAD) coronary artery as well as using great saphenous vein (GSV) grafts to revascularize other coronary arteries. A Medtronic stabilizer was used together with pericardial stitches for better visualization of the anastomotic site. Intra-coronary shunts were used in addition to humid air blower with normal saline for clearing of the operative field.

Postoperative outcomes: The clinical outcomes of our study were the occurrence of low cardiac output (LCO) defined by the
Table 2. Postoperative adverse outcomes

\begin{tabular}{lc}
\hline Postoperative adverse outcomes & Count (\%) \\
\hline Low CO & $5(7.6 \%)$ \\
Postoperative AF & $4(6 \%)$ \\
Perioperative MI & $4(6 \%)$ \\
Prolonged ventilation & $3(4.6 \%)$ \\
Mortality & $2(3 \%)$
\end{tabular}

need for inotropic support in the form of adrenaline $>100 \mathrm{ng} /$ $\mathrm{kg} / \mathrm{min}$ or dobutamine $>20 \mathrm{mcg} / \mathrm{kg} / \mathrm{min}$ at any time postoperatively and/or the need for intra-aortic balloon pump, postoperative $\mathrm{AF}$, postoperative myocardial infarction (MI) evidenced by cardiac enzymes and electrocardiogram (ECG), postoperative prolonged mechanical ventilation defined by intubation and mechanical ventilation for more than 24 hours postoperatively and/or re-intubation, as well as the lengths of ICU and hospital stays, and in-hospital mortality.

Statistical analysis: An Excel spreadsheet was established for the entry of data. We used validation checks on numerical variables and option-based data entry method for categorical variables to reduce potential errors. Data were coded and entered using SPSS (Statistical Package for the Social Sciences) version 24. Data were presented as mean, standard deviation and interquartile range in quantitative data as well as fre-quency (count) and relative frequency (percentage) for categorical data. Comparisons between quantitative variables were done using the nonparametric Mann-Whitney test. Correlations between quantitative variables were done using spearman correlation coefficient. ROC curve was constructed with area under the curve (AUC) to detect the best cut-off value of NTproBNP for detection of outcomes. $P$-values less than 0.05 were considered statistically significant.

\section{RESULTS}

Preoperative data: Sixty-five patients were recruited in this study. The mean age was $57.62 \pm 7.21$ years. There were 56 males $(86.15 \%)$. Ten patients $(15.38 \%)$ had diabetes mellitus (DM), 42 patients (64.62\%) were hypertensive, and only one patient suffered from a $70 \%$ stenosis of the right carotid artery. The mean preoperative EF of patients was $50.91 \pm$ 8.13, while the mean calculated EuroscoreII was $1.76 \pm 0.34$ (range 0.55-1.82). The mean preoperative NTproBNP levels were $312.41 \pm 329.93 \mathrm{pg} / \mathrm{mL}$ (range 80-397.5) (Table 1).

Operative data: The mean length of operations was $2.9 \pm 1.1$ hours, and the mean number of performed grafts was $3.2 \pm 0.9$.

Postoperative outcomes: Only two patients died (3\%); one due to sepsis and the other due to respiratory failure. Three patients required prolonged mechanical ventilation (4.6\%); one due to neurological insult in the form of minor brain stem embolic infarction (the only patient suffering from such complication), with the other two due to chest infection. Four patients $(6 \%)$ suffered from new onset postoperative 
Table 3. Relation between preoperative NTproBNP levels and postoperative adverse outcomes

\begin{tabular}{lccc}
\hline & \multicolumn{4}{c}{$\begin{array}{c}\text { Preoperative NTproBNP } \\
(\mathrm{pg} / \mathrm{mL})\end{array}$} \\
& No mean \pm SD & \\
Postoperative adverse outcomes & Yes & $P$ \\
\cline { 2 - 4 } Low cardiac output syndrome & $296.84 \pm 329.75$ & $490 \pm 307.97$ & .168 \\
AF & $306.37 \pm 333.77$ & $400 \pm 292.91$ & .462 \\
Perioperative MI & $303.79 \pm 331.23$ & $437.5 \pm 326.22$ & .397 \\
Prolonged ventilation & $300.33 \pm 330.69$ & $550 \pm 244.33$ & .121 \\
Mortality & $306.33 \pm 331.15$ & $495 \pm 318.19$ & .306
\end{tabular}

AF; three during their ICU stay and one after discharge to the in-patient ward. All cases with postoperative AF successfully were converted to sinus rhythm by means of amiodarone infusion. Five patients $(7.6 \%)$ had LCO, of which three needed IABP, and four patients $(6 \%)$ had postoperative myocardial infarction evidenced by elevated cardiac enzymes. The mean ICU stay was $3.37 \pm 0.84$ days, and mean hospital stay was $6.38 \pm 1.3$ days (Table 2).

There were no significant differences in preoperative NTproBNP levels in patients who had or didn't have any of the postoperative complications or mortality (Table 3).

In addition, preoperative NTproBNP didn't correlate with duration of ICU stay or postoperative hospital stay $(P>.05)$.

\section{DISCUSSION}

Studies investigating the value of perioperative BNP and NTproBNP levels in predicting the postoperative outcomes of cardiac surgery are very heterogeneous regarding the peptide used, the time and frequency of sampling, the preoperative co-morbidities, the clinical endpoints, the duration of follow up, and results. In our study, we have chosen NTproBNP (and not BNP) since the former is more biochemically stable than BNP. Samples for measuring NTproBNP can be withdrawn into glass or plastic tubes and do not require an addition of protease inhibitors, such as EDTA. Also, the in-vivo day-to-day biologic variation in HF patients is about $38 \%$ for BNP and 28\% for NTproBNP [Gaggin 2013]. In addition, we excluded from our study patients with preoperative factors that might influence not only preoperative NTproBNP levels but also the postoperative outcomes. This also helped in obtaining a homogenous study population. Thus, severe chronic obstructive pulmonary disease, renal impairment, ejection fraction $<40 \%$, preoperative atrial fibrillation, NYHA III/IV and associated valve lesions necessitating surgical intervention were all among the exclusion criteria. The fact that inclusion of valve surgery can dramatically alter the prognostic performance of preopreative natriuretic peptide is most clearly shown by the study done by Fellahi et al [Fellahi 2012], which included CABG and aortic valve replacement (AVR) patients. In this study, ROC curve analysis of preoperative BNP levels revealed an AUC of $0.67(P=.002)$ for predicting major adverse cardiac events (MACEs). However, when they reanalyzed the data after dividing the patients into two groups, the CABG group and AVR group, preoperative BNP levels were more accurate in predicting MACEs after AVR (area under ROC curve $=0.78, P<.001$ ) and not after CABG (area under ROC curve $=0.54, P=.32$ ).

Our study showed no significant differences in preoperative NTproBNP levels between patients with or without postoperative myocardial infarction. This is similar to the reports by Eliasdottir et al [Elíasdóttir 2008], Schachner et al [Schachner 2010], and Akhmedova et al [Akhmedova 2020]. Attaran et al [Attaran 2009] also showed similar results but they used BNP in their study. Postoperative myocardial infarction is more likely caused by intra-operative influences and thus is not captured by preoperative natriuretic peptide levels.

Our study showed no significant differences in NTproBNP between patients with or without postoperative AF. This is similar to the reports of Jogia et al [Jogia 2007] and Attaran et al [Attaran 2009]. On the other hand, Cuthbertson et al [Cuthbertson 2009] reported an odds ratio of $1.02(95 \%$ confidence interval $=1-1.03$ ) per $250 \mathrm{pg} / \mathrm{mL}$ increase in preoperative NTproBNP levels and the development of postoperative $\mathrm{AF}(P=.02)$. However apart from the fact that the $P$-value itself is of poor predictive performance, a $95 \%$ confidence interval of 1 undermines its statistical significance. Noteworthy is the incidence of postoperative $\mathrm{AF}$ in our study (6\%), which is obviously lower than that included in other studies; reporting an incidence up to $35 \%$. This is attributed to the lower incidence of risk factors found in our patient population, especially preoperative $\mathrm{AF}$, significant valvular diseases, low EF, and severe COPD. In addition, all our patients received their beta-blockers until the morning of surgery and resumed them on the second postoperative day. In fact, Chen et al [Chen 2013] reported a similar incidence of new onset arrhythmias (3/76 patients, $3.9 \%$ ) with a population similar to ours.

Our study showed no significant differences in NTproBNP levels in patients, who did or didn't develop postoperative LCO, which we considered as the need for inotropic support in the form of adrenaline $>100 \mathrm{ng} / \mathrm{kg} / \mathrm{min}$ or dobutamine $>20 \mathrm{mcg} / \mathrm{kg} / \mathrm{min}$ at any time postoperatively and $/$ or the need for intra-aortic balloon pump. This also was the case in the study reported by Öztekin et al [Öztekin 2017]. In contrast, Eliasdottir et al [Elíasdóttir 2008], Akhmedova et al [Akhmedova 2020], and Krzych et al [Krzych 2011] reported that NTproBNP levels predicted inotropic support at cut-off points of $376 \mathrm{pg} / \mathrm{mL}, 430 \mathrm{pg} / \mathrm{mL}$ and $684 \mathrm{pg} / \mathrm{mL}$, respectively. This might make sense due to the fact that the mean preoperative NTproBNP levels in our study was $312.41 \pm 329.93$ $\mathrm{pg} / \mathrm{mL}$, which is lower than the cut-off points in these studies.

Our study showed no significant differences in preoperative NTproBNP levels in patients who required (or not) prolonged mechanical ventilation for $>24 \mathrm{hr}$. Similarly, Öztekin et al [Öztekin 2017] in 2017, Sindhvananda et al [Sindhvananda 2019] in 2019, and Akhmedova et al [Akhmedova 2020] in 2020 found no significant differences in ventilation time in patients with high versus low preoperative NTproBNP levels. This is in contrast to Jogia et al [Jogia 
2007] in 2007, Cuthbertson et al [Cuthbertson 2009] in 2009, and Liu et al [Liu 2013] in 2013, who reported low to modest correlations between preoperative NTproBNP levels and the need for postoperative mechanical ventilation for $>24 \mathrm{hr}$. Thus, earlier studies showed significant, but weak correlation between preoperative natriuretic peptides and ventilation time, while more recent studies lack that finding. One can speculate that since the correlation was weak to begin with, even minor improvements in mechanical ventilation technologies and protocols might have rendered it invalid.

Our study showed no significant correlation between preoperative NTproBNP levels and ICU stay $(3.37 \pm 0.84$ days). Similar findings were obtained by Chen et al [Chen 2013], Öztekin et al [Öztekin 2017], and Akhmedova et al [Akhmedova 2020] who found no significant relation between preoperative BNP and NTproBNP levels and ICU stay for $>4$ days. On the other hand, Fellahi et al [Fellahi 2011], Jogia et al [Jogia 2007], and Cuthbertson et al [Cuthbertson 2009] found a limited diagnostic performance of preoperative BNP levels to predict a prolonged ICU stay. However, Jogia et al [Jogia 2007] reported a mean ICU stay of $27 \pm 18 \mathrm{hr}$, while Cuthbertson et al [Cuthbertson 2009] defined prolonged ICU stay as $>$ one day. By contrast, our policy is to keep patients routinely in the ICU for at least two postoperative days. In addition, these studies [Jogia 2007; Cuthbertson 2009; Fellahi 2011] included patients with $\mathrm{EF}<35 \%$, NYHA III/IV and valve surgery patients.

Similar to our study, Öztekin et al [Öztekin 2017] found no significant difference between patients with low and high levels of preoperative NTproBNP, in terms of the duration of hospital stay. Chen's study [Chen 2013], which is similar to ours in cohort and outcome, reported higher but non-significant preoperative NTproBNP levels with prolonged hospitalization.

Our study showed no significant correlation between preoperative NtproBNP levels and mortality (2/65 patients). This is similar to Jogia et al [Jogia 2007] who, as in our study, reported low mortality (2/118 patients). Recently, Brynildsen et al [Brynildsen 2018] found that preoperative NTproBNP level of $>1170 \mathrm{pg} / \mathrm{mL}$ predicted mortality with a sensitivity of $66 \%$, specificity of $73 \%$ and area under ROC curve of 0.73 , while EuroSCORE II had an area under ROC curve of 0.74 . Also, Suc et al [Suc 2020] found a prediction of preoperative BNP level to mortality with an area under ROC curve of 0.66 compared to EuroSCORE II, which had an area under ROC curve of 0.8 .

Our study had the strength points of a prospective research with a homogenous cohort of low-risk patients registered for elective OPCAB surgeries to eliminate other influences on preoperative NTproBNP level as a predictor of adverse postoperative outcomes and in-hospital mortality. Therefore, the absence of significant correlation between preoperative NTproBNP level and the adverse postoperative outcomes as well as in-hospital mortality in our study is most likely due to the low incidence of complications and low preoperative NTproBNP level secondary to the low preoperative risk factors. This finding questions the sensitivity of preoperative NTproBNP as a predictor of poor outcome after off-pump
CABG when in relative low levels such as in our study (312.41 $\pm 329.93 \mathrm{pg} / \mathrm{mL}$ ). Thus, whether preoperative natriuretic peptides are significant reliable predictors of poor outcome or not will remain an interesting research question necessitating more prospective specific studies.

\section{CONCLUSION}

Our study showed no significant correlation between preoperative NTproBNP levels and postoperative LCO, atrial fibrillation, postoperative myocardial infarction, lengths of ICU and hospital stays, prolonged mechanical ventilation, as well as in-hospital mortality following elective off-pump CABG. Therefore, more prospective specific studies are needed to delineate the role of preoperative natriuretic peptides as significant sensitive predictors of poor outcome after CABG surgery.

\section{REFERENCES}

Akhmedova IA, Kudaiberdiev TZ, Abibillaev DA, et al. 2020. Relationship of preoperative NT-pro-BNP with clinical, perioperative and prognostic markers in cardiacsurgery: Preliminary study results. Heart Vessels Transplant. 4:99-110.

Attaran S, Sherwood R, Desai J, Langworthy R, Mhandu P, John L, ElGamel A. 2009. Brain natriuretic peptide a predictive marker in cardiac surgery. 9:662-666.

Brynildsen J, Petäjä L, Pettilä V, et al. 2018. The predictive value of NTproBNP and hs-TnT for risk of death in cardiac surgical patients. Clinical Biochemistry. 53:65-71.

Chen TH, Lin CL, Shih JJ, et al. 2013. Plasma B-type natriuretic peptide in predicting outcomes of elective coronary artery bypass surgery. Kaohsiung Journal of Medical Sciences. 29:254-258.

Cuthbertson BH, Croal BL, Rae D, et al. 2009. N-terminal pro-B-type natriuretic peptide levels and early outcome after cardiac surgery: a prospective cohort study. Br J Anaesth. 103:647-653.

Elíasdóttir SB, Klemenzson G, Torfason B, Valsson F. 2008. Brian natriuretic peptide is a good predictor for outcome in cardiac surgery. Acta Anaesthesiol Scan. 52:182-187.

Emdin M, Passino C, Prontera C, et al. 2004. Cardiac natriuretic hormones, neuroor-mones, thyroid hormones and cytokines in normal subjects and patients with heart failure. Clin Chem Lab Med. 42:627-636.

Fellahi JL, Daccache G, Makroum Y, Massetti M, Gérard JL, Hanouz JL. 2012. The Prognostic Value of B-Type Natriuretic Peptide After Cardiac Surgery: A Comparative Study Between Coronary Artery Bypass Graft Surgery and Aortic Valve Replacement.Journal of Cardiothoracic and Vascular Anesthesia. 26:624-30.

Fellahi JL, Daccache G, Rubes D, Massetti M, Gérard JL, Hanouz JL. 2011. Does Preoperative B-Type Natriuretic Peptide Better Predict Adverse Outcome and Pro-longed Length of Stay Than the Standard European System for Cardiac Operative Risk Evaluation After Cardiac Surgery? J Cardiothorac Vasc Anesth. 25:256-262.

Gaggin and Januzzi. 2013. Biomarkers and diagnostics in heart failure. Biochem Bio-phys Acta. 1832:2442-245.

Gaggin and Januzzi J. 2014. Natriuretic peptides in heart failure 
syndrome. Clin Lab Med. 34:43-58.

Iwanaga Y, Nishi I, Furuichi S, et al. 2006. B-type natriuretic peptide strongly reflects diastolic wall stress in patients with chronic heart failure: comparison between sys-tolic and diastolic heart failure. Journal of American College of Cardiology. 47:742-748.

Jogia PM., Kalkoff M, Sleigh JW, et al. 2007. NTproBNP secretion and clinical end-points in cardiac surgery intensive care patients. Anaesth Intensive Care. 35:363-369.

Krzych Ł, Szurlej D, Kołodziej T. 2011. Diagnostic accuracy of preoperative NT-proBNP level in predicting short-term outcomes in coronary surgery: a pilot study. Kardiologia Polska. 69:1121-1127.

Litton E and Ho KM. 2012. The use of pre-operative brain natriuretic peptides as a predictor of adverse outcomes after cardiac surgery: a systematic review and meta-analysis. European Journal of Cardio-thoracic Surgery. 41:525-534.

Liu H, Wang C, Liu L, Zhuang Y, Yang X, Zhang Y. 2013. Perioperative application of $\mathrm{N}$-terminal pro-brain natriuretic peptide in patients undergoing cardiac surgery. Journal of Cardiothoracic Surgery. 8:1-5.

Öztekin A, Memetoðlu M, Kutlu R, et al. 2017. The Predictive Value of Preoperative Serum NT-proBNP Levels for the Need for Inotropic Supportin the Postoperative Pe-riod in Patients Undergoing Coronary Artery Bypass Grafting. Cardiovasc. j 9:90-96.

Schachner T, Wiedemann D, Fetz H, Laufer G, Kocher A, Bonaros N. 2010. Influence of preoperative serum $\mathrm{N}$-terminal pro-brain type natriuretic peptide on the postoperative outcome and survival rates of coronary artery bypass patients. Clinics. 65:1239-1245.

Sindhvananda W, Bunpeth C, Chareonkulnawanun N. 2019. N-Terminal Pro-Brain NatriureticPeptide in Post Cardiac Surgery as a Predictor of Ventilator-Weaning Outcomes. Int J AnestheticAnesthesiol. 6:085.

Suc G, Estagnasie P, Brusset A, Procopi N, Squara P, Nguyen LS. 2020. Effect of BNP on risk assessment in cardiac surgery patients, in addition to EuroScore II. Scientific Reports. 10:10865. 